\title{
EDITORIAL
}

\section{A national health care data network is overdue}

\author{
Suzanne N. Morin MD MSc, Ken Flegel MDCM MSc
}

Cite as: CMAJ 2017 July 24;189:E951. doi: 10.1503/cmaj.170767

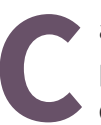
anada has administrative health care data of world-class potential. Our publicly funded system covers the entire and diverse population, there is a set of single third-party payers, and care is provided by similar facilities in each province and territory. Health data repositories exist across the country, but none are truly national or as comprehensive as they could be. Should we choose to, these repositories could be integrated into a national network that would enable us to measure relevant health indicators consistently and efficiently at the national, provincial and local levels. Such a rich data bank could contribute to our understanding of the burden and management of diseases, support public health measures and allow for wiser resource allocation.

Provincial and territorial health authorities systematically collect detailed information on prescribed medications, physician encounters and hospital admissions. Health data are collected in multiple forms, such as electronic health records, digital health-monitoring devices, registries and cohorts, and in non-health-related data repositories. We have a near-national organization in the Canadian Institute for Health Information, but Quebec does not systematically contribute to it. Certain provincial entities have also created comprehensive health care data repositories that include linking administrative data to clinical registries.

Currently, these data are not readily accessible to researchers or policy-makers nationally. Existing regional infrastructures are complex, and data-sharing agreements and standardization of data collection among jurisdictions are lacking. Even when these barriers are surpassed, using these data is difficult, owing to formidable time delays in gaining access in certain provinces.

Creating a comprehensive, coherent and readily accessible national data bank could have many benefits. ${ }^{1}$ Understanding the burden of disease, locally to nationally, would enable rational health care planning and appropriate distribution of resources. Health costs are likely to decrease from such advances. ${ }^{2}$

Huge benefits could also be expected from more effective health care research activity. Data linkage across databases will provide richer data sets, with potential for new multidisciplinary collaborations for health research initiatives. Expensive and timeconsuming efforts to gain access to representative samples of the population would be much reduced. Research on the entire population and across jurisdictions would be enhanced and provide real-world data, and research on rare diseases or special populations would become more feasible.

Other countries, such as Australia and the United Kingdom, have implemented comprehensive national networks that link multiple large data sets across jurisdictions. These receive national funding and contribute to the public health and patient care agendas. National networks, such as the Canadian Network for Observational Drug Effect Studies and the chronic disease surveillance program from Health Canada, do exist in Canada, but have restricted mandates.

Teams of investigators have been working on plans to develop a pan-Canadian data network that would support effective use of linkable data holdings across the country. Using available health data sets, the investigators propose developing algorithms that identify specific health conditions within the data holdings, harmonizing common data across jurisdictions and creating shared analytic protocols. This would allow data to be analyzed within each jurisdiction and summary data to be distributed in a safe manner through this network, including use of regulated agreements to protect every citizen's right to privacy. ${ }^{3}$

The Canadian Institutes of Health Research, with its strategy to have patient-oriented research with data support units in each provincial and territorial jurisdiction, should be an ideal and timely partner in such initiatives.

A national health care data set is merely a dream now, but it is a dream that is too important to leave solely to the politicians. All who have a stake in health care delivery should advocate for such a resource. The nation's health will surely benefit from timely and further-reaching access to comprehensive health care information - a fitting present for Canada's 150th birthday.

\section{References}

1. Benefits of accessing and linking data for health research. In: Accessing health and health-related data in Canada. Ottawa: The Expert Panel on Timely Access to Health and Social Data for Health Research and Health System Innovation, Council of Canadian Academies; 2015:50-3.

2. Accessing health and health-related data: benefits, risks, and barriers. In: Accessing health and health-related data in Canada. Ottawa: The Expert Panel on Timely Access to Health and Social Data for Health Research and Health System Innovation, Council of Canadian Academies; 2015:48-9.

3. Accessing data for research purposes: Canada's current legal and ethical framework. In: Accessing health and health-related data in Canada. Ottawa: The Expert Panel on Timely Access to Health and Social Data for Health Research and Health System Innovation, Council of Canadian Academies;2015:76-84.

Competing interests: For Ken Flegel, see www.cmaj.ca/site/misc/ cmaj_staff.xhtml. Suzanne Morin reports grants from Merck and from Amgen, outside the submitted work

Affiliations: Department of Medicine, McGill University, Montréal, Que. (Morin, Flegel); Senior Editor (Flegel), CMAJ

Correspondence to: pubs@cmaj.ca 